\title{
Session 6: Molecular Biology II
}

\author{
Tuesday 8th November 2011. Moderator: Don Capra
}

[10.40-11.00]

'Tailored antibody library design for optimized drug development'

Robert Rauchenberger

MorphoSys AG, Martinsreid/Planegg, Germany

One key feature of MorphoSys' core technology, the fully human antibody library HuCAL ${ }^{\circledR}$ ( Combinatorial Antibody Library), is a CDR design which mimics the natural repertoire. Its diversity is realized using MorphoSys' proprietary TRIM-technology. Recently, also the world leading technology in gene synthesis, Slonomics ${ }^{\circledR}$ became part of MorphoSys' technology portfolio. Slonomics ${ }^{\circledR}$ represents a fully automated genetic engineering platform that utilizes sets of double stranded DNA triplets in the controlled fabrication of highly diverse combinatorial gene libraries with unprecedented speed. The unique combination of MorphoSys' library design, antibody selection, screening and development know-how with Slonomics ${ }^{\circledR}$ gives rise to the new antibody optimization platform, arYla ${ }^{\mathrm{TM}}$. Examples of highly efficient affinity maturation and successful reduction of hot spots for unwanted posttranslational modifications will be presented as well as screening strategies for improved thermostability and increased expression rates.

\section{[11.00-11.20]}

'Novel human antibody platforms based on genetically engineered rats'

Roland Buelow

OMT Inc., Palo Alto, CA, USA

Open Monoclonal Technology, Inc. (“OMT”, www. omtinc.net) is a private biotechnology company that developed a new, fully human monoclonal antibody platform. Many companies would like to develop new monoclonal antibodies, but available technologies are expensive, and potential targets often are limited by exclusivity agreements. OMT's antibody platform is available for all targets, has broad freedom to operate, and uses technology protected by patents.

OMT's platform is based on transgenic rats. The rat is a widely used laboratory animal with a well characterized immune system, a nearly complete genome sequence, and established transgenesis and hybridoma technologies. OMT's genetic engineering approach is the result of an improved understanding of B-cell development and a novel approach to the inactivation of endogenous antibody expression. OMT developed a new zinc-finger-nuclease-mediated technique to generate immunoglobulin knockout rats. Analysis of protein expression in serum demonstrated inactivation of rat heavy and light chain immunoglobulin expression in homozygous knockout animals. To eliminate suboptimal B-cell receptor signaling, OMT designed immunoglobulin loci including human antibody genes that allow proper assembly of the B-cell receptor complex, normal signaling and high expression of human antibodies. Immunoglobulin transgenes, encoding human kappa or lambda light chains, and a heavy chain were introduced into the rat genome using conventional embryo microinjection. Breeding of immunoglobulin transgenic and knock-out animals resulted in animals expressing a diversified repertoire of antibodies with fully human idiotypes.

[11.20-11.40]

'Engineering $\mathrm{CHO}$ cell line for enhanced production of $\mathbf{m A b s}$ and Fc:fusion proteins'

Pierre-Alain Girod

Selexis, Plan-les-Ouates/Geneva, Switzerland

Abstract not provided. 
[11.40-12.00]

'Using thermostabilised receptors (StaRs) to generate therapeutic antibodies to GPCR targets'

Catherine Hutchings

Heptares Therapeutics Ltd, Welwyn Garden Ciry, UK

G-protein couple receptors (GPCRs) have historically been considered as difficult targets for antibody development, but are one of the most important classes of drug targets. This presentation describes the opportunity for targeting GPCRs with antibodies and describes examples of therapeutic GPCR antibodies currently in clinical development.

GPCRs are implicated in a wide variety of diseases where antibody therapeutics are currently used. These include inflammatory diseases, such as rheumatoid arthritis and Crohn's disease, as well as metabolic disease and cancer. Raising antibodies to GPCRs has been very difficult due to problems in obtaining suitable antigen, since GPCRs are often expressed a low levels in cells and are very unstable when purified. Hence, there has been a fairly limited degree of success with generating anti-GPCR antibodies to date.

There are many technical hurdles to overcome in raising antibodies to membrane proteins, such as GPCRs, however developments in stabilising receptors using mutagenesis or additives to formulate stable pure protein, as well as methods to over-express receptors in cell systems are contributing to the growing interest in targeting GPCRs with antibodies. Here we present a novel approach using Stabilised Receptors (StaRs), where GPCRs have been engineered to include a small number of point mutations that greatly increases the thermostability of the receptors.

This permits generation of a GPCR that is sufficiently stable for large scale production and purification of milligram quantities of homogenous material. This approach has been used for a diverse set of GPCRs.

The thermostabilization process pushes the receptor into a specific chosen conformational state, for example, agonist or antagonist. This may present the immune system with conformationally specific epitopes, thereby allowing the selection of antibodies that will selectivity bind to and stabilize specific conformations. This emerging technology has been employed in both in vitro and in vivo approaches to demonstrating its potential to generate therapeutic antibodies.
[12.00-12.30]

'Bioactive antibody peptides'

Luciano Polonelli

Universita degli Studi di Parma, Parma, Italy

Antibodies (Abs) are increasingly seen as important for novel antigen-driven drugs in diverse clinical settings, including infectious diseases and oncology. Abs, however, elicit in the treated host an anti-Ab response, resulting in adverse reactions which may limit their therapeutic efficacy. The modular domain architecture of Abs has been exploited to generate alternative reduced formats, essentially devoid of the $\mathrm{Fc}$ region. Paradigmatic studies on single chain fragment variable region Abs mimicking the wide-spectrum antimicrobial activity of a yeast killer toxin led to the proof of concept that, irrespective of the specificity and the class of the native $\mathrm{Ab}$, peptides belonging either to the complementarity determining or constant region, may display differential in vitro, ex vivo and/or in vivo antimicrobial (Candida albicans), antiviral (HIV-1), antitumor (melanoma) and modulatory (immune cells) activities, conceivably mediated by different mechanisms of action. Alanine substitution of single residues of $\mathrm{Ab}$ peptides, used as surrogates of natural point mutations, resulted in further differential increased, unaltered or decreased biological activities. It is hypothesized that $\mathrm{Ab}$ peptides occurring beyond the half life of Abs, potentially diversified by somatic mutation and clonal selection by antigens, may represent a link between adaptive and innate immunity. Effective delivery is a critical issue in the use of conventional free drugs. Studies on the structure-function relationship of some therapeutic $\mathrm{Ab}$ peptides revealed their ability to spontaneously and reversibly self-assemble in an organized network of fibril-like structures. While the self-assembled state may provide protection against proteases, the slow kinetic of dissociation assures a release of the active form over time. The high rate of bioactive fragments and recent advances in peptide delivery, stability and design suggest that Abs may represent unlimited sources for the search of new antiinfectious and antitumor agents. 